\title{
Overproduction of intestinal lipoprotein containing apolipoprotein B-48 in Psammomys obesus: impact of dietary $n-3$ fatty acids
}

\author{
E. Levy $\cdot$ S. Spahis $\cdot$ E. Ziv $\cdot$ A. Marette $\cdot$ M. Elchebly $\cdot$ \\ M. Lambert • E. Delvin
}

Received: 28 December 2005 / Accepted: 19 April 2006 / Published online: 21 June 2006

(C) Springer-Verlag 2006

\begin{abstract}
Aims/hypothesis Emerging evidence underscores the important role of the small intestine in the pathogenesis of dyslipidaemia in insulin resistance and type 2 diabetes. We therefore tested the hypothesis that $n-3$ fatty acids improve
\end{abstract}

E. Levy $\cdot$ S. Spahis

Department of Nutrition, Research Centre, Ste-Justine Hospital and University of Montréal,

Montreal, QC, Canada

E. Ziv

Diabetes Unit, Division of Internal Medicine,

Hadassah University Hospital,

Jerusalem, Israel

\section{A. Marette}

Department of Physiology and Lipid Research Unit, Laval University Hospital Research Center,

Ste-Foy, QC, Canada

\section{Elchebly}

Department of Endocrinology, Research Centre,

Ste-Justine Hospital and University of Montréal,

Montreal, QC, Canada

M. Lambert

Department of Paediatrics, Research Centre,

Ste-Justine Hospital and University of Montréal,

Montreal, QC, Canada

E. Delvin

Department of Biochemistry, Research Centre,

Ste-Justine Hospital and University of Montréal,

Montreal, QC, Canada

E. Levy $(\square)$

CHU-Sainte-Justine,

3175 Côte Ste-Catherine Road,

Montreal, QC H3T 1C5, Canada

e-mail: emile.levy@recherche-ste-justine.qc.ca the various events governing intra-enterocyte lipid transport in Psammomys obesus gerbils, a model of nutritionally induced metabolic syndrome.

Materials and methods Experiments were carried out on Psammomys obesus gerbils that were assigned to an isocaloric control diet and a diet rich in fish oil for 6 weeks. Results Increased dietary intake of fish oil lowered body weight and improved hyperglycaemia and hyperinsulinaemia. It simultaneously decreased de novo intestinal lipogenesis and lipid esterification of the major lipid classes, e.g. triglycerides, phospholipids and cholesteryl esters, particularly in insulinresistant and diabetic animals. Accordingly, lessened activity of monoacylglycerol and diacylglycerol acyltransferase was recorded. As assessed in cultured jejunal explants incubated with either $\left[{ }^{14} \mathrm{C}\right]$-oleic acid or $\left[{ }^{35} \mathrm{~S}\right]$-methionine, fish oil feeding resulted in diminished triglyceride-rich lipoprotein assembly and apolipoprotein (apo) B-48 biogenesis, respectively. The mechanisms did not involve apo B-48 transcription or alter the gene expression and activity of the critical microsomal triglyceride transfer protein. Rather, the suppressed production of apo B-48 by $n-3$ fatty acids was associated with intracellular proteasome-mediated posttranslational downregulation in insulin-resistant and diabetic animals.

Conclusions/interpretation Our data highlight the beneficial impact of $n-3$ fatty acids on adverse effects of the metabolic syndrome and emphasise their influence on intestinal lipid transport, an effect which may limit postprandial lipaemia and the risk of atherosclerosis.

Keywords Apo B-48 · DGAT · Gerbil · Intestinal fat absorption $\cdot$ MGAT $\cdot$ Microsomal triglyceride transfer protein $\cdot n-3$ fatty acids $\cdot$ Psammomys obesus .

TG-rich lipoprotein 


\section{Abbreviations}

apo apolipoprotein

DGAT diacylglycerol acyltransferase

FA fatty acids

LC-PUFA long chain polyunsaturated fatty acids

MGAT monoacylglycerol acyltransferase

MTP microsomal triglyceride transfer protein

\section{Introduction}

Hypertriglyceridaemia is important in the genesis of cardiovascular disease. It is now well established that the risk of developing coronary heart disease risk is positively associated with increasing levels of triglycerides [1-4]. It has repeatedly been confirmed that triglycerides are an independent predictor of cardiovascular disease, e.g. by a huge meta-analysis [5], as well as by intervention [6] and observational long-term [1] studies. In addition, postprandial hyperlipidaemia has been linked to atherosclerosis, even in fasting normocholesterolaemic subjects [7-10].

Dyslipidaemia is common in patients with insulin resistance and type 2 diabetes, and is thought to be responsible for a considerable proportion of morbidity and mortality related to cardiovascular disease $[11,12]$. Especially, hypertriglyceridaemia is among the powerful risk factors for atherosclerosis in patients with type 2 diabetes or impaired glucose tolerance, conditions which are characterised by increased VLDL secretion from the liver [1, 13]. The enhanced mobilisation of NEFA from the adipose tissue augmented the rate of hepatic NEFA uptake and stimulated the secretion of apolipoprotein (apo) B-100, leading to increased numbers of apo-B-containing particles and possibly hypertriglyceridaemia [14]. Although fasting triglycerides are considered important determinants of coronary heart and/or carotid artery disease in people with diabetes, the progression of coronary lesions over 5 years was related to postprandial lipaemia [8]. The response of plasma triglycerides to a standard fat load is much greater in type 2 diabetic than in non-diabetic subjects $[15,16]$, and is correlated with intima media thickness, suggesting that prolonged exposure of the arterial wall to triglyceride-rich chylomicron remnants accelerated the atherogenic process. Relatively little attention has been given to the mechanisms behind postprandial triglycerides. However, the raised fasting triglyceride-rich lipoproteins often found in diabetes and associated with apo B-48 may be derived from increased intestinal chylomicron production $[17,18]$. According to these recent studies, synthesis rather than clearance may be the major cause of the increase in atherogenic postprandial particles in people with insulin resistance and type 2 diabetes.
Fish oil containing $n$-3 fatty acids (FA) has shown protective effects against atherosclerotic diseases since it decreased serum lipid concentrations, had antithrombotic and anti-inflammatory effects, lowered blood viscosity, and reduced blood pressure [19-21]. Fish oil also improved dyslipidaemia, hypertension and endothelial function in subjects with diabetes $[22,23]$. However, intake of $n-3$ FA has been associated with impaired glycaemic control, leading both to hyperglycaemia and insulin resistance [24, 25]. In view of these inconsistent studies, proof that $n-3$ long chain polyunsaturated fatty acids (LC-PUFA) can prevent subjects with insulin resistance or impaired glucose tolerance from developing type 2 diabetes remains to be established. Direct evidence linking $n-3$ FA to favourable effects on intestinal lipid transport is also lacking. In the present study, we examined the influence of $n-3$ FA on the development of insulin-resistant and type 2 diabetic states, as well as on intestinal oversecretion of apo B-48 lipoproteins in Psammomys obesus gerbils, an excellent animal model for the investigation of insulin resistance and type 2 diabetes.

\section{Materials and methods}

Experimental animals and diet

Experiments were carried out on Psammomys obesus gerbils purchased from Hebrew University Animal Breeding Facility (Jerusalem, Israel). Upon weaning at 3 weeks of age, the animals were randomly assigned to two isocaloric diets $(2.93 \mathrm{kcal} / \mathrm{g}$ body weight $)$ for 6 weeks, which differed only in lipid composition and contained (in $\mathrm{g} / \mathrm{kg}$ ): casein, 225; cornstarch, 446; sucrose, 223; cellulose, 31; dl-methionine, 1; mineral mix, 14; vitamin mix, 10; oil 50. One diet, considered as control, contained lard as the major lipid source together with corn oil $(1.9 \mathrm{~g} / 100 \mathrm{~g}$ diet $)$; the second experimental diet was also lard/corn-oil-based, but $7 \%$ of the lard/oil was replaced with sardine oil and was named $n-3$ FA diet. Chow was changed daily and contained $t$-butylhydroquinone in order to prevent peroxidation. The animals were housed singly in polypropylene cages at a constant temperature of 22 to $23^{\circ} \mathrm{C}$ in a humidity-controlled animal facility with a 12-h light/dark cycle. Free access to water and food was provided. Before being killed, Psammomys obesus gerbils were monitored for body weight and tail blood concentrations. Animals were anaesthetised with ketamine hydrochloride and blood was drawn from the aorta. Animals were classified, as in our previous studies [26], into three groups according to plasma glucose and insulin concentrations: (1) normoglycaemia/ normoinsulinaemia (Group A); (2) normoglycaemia/hyperinsulinaemia (Group B); and (3) hyperglycaemia/hyper- 
insulinaemia (Group C). The distribution into classes is defined on the basis of plasma glucose and insulin values as follows: Group A, glucose $<5.5 \mathrm{mmol} / 1$, insulin $<100 \mathrm{mU} / 1$; Group B, glucose $<5.5 \mathrm{mmol} / 1$, insulin $>100 \mathrm{mU} / \mathrm{l}$; Group C, glucose $>5.5 \mathrm{mmol} / \mathrm{l}$, insulin $>100 \mathrm{mU} / 1$. In Group B, glucose underutilisation and/or overproduction was compensated by hyperinsulinaemia so that animals remained normoglycaemic, but gained adipose tissue. In Group C, the animals were hyperglycaemic despite extreme hyperinsulinaemia. This classification was consistently validated by various techniques, such as glucose tolerance tests, measurement of insulin secretion and euglycaemic-hyperinsulinaemic clamp. Finally, as noted for humans, the levels of glycaemia $2 \mathrm{~h}$ after food or glucose load were 5.5 to $6.7 \mathrm{mmol} / 1$ in Group A and 10.0 to $11.1 \mathrm{mmol} / 1$ in Group $\mathrm{C}$, whereas insulin concentrations were always under $100 \mathrm{mU} / 1$ in Group A and between 100 and $150 \mathrm{mU} / \mathrm{l}$ in Group C. Group B was always normoglycaemic and hyperinsulinaemic. All procedures were performed in accordance with the animal experimentation guidelines of Sainte-Justine Hospital.

Intestinal organ cultures

The jejunum from Psammomys obesus gerbils was cleared of mesentery, split longitudinally, washed in culture medium, and cut into explants $(3 \times 7 \mathrm{~mm})$. Five to seven explants were randomly transferred on to lens paper, with the mucosal side facing up in each organ culture dish (Falcon Plastics, Los Angeles, CA, USA). Six dishes were used for each experimental condition. An amount of medium $(0.8 \mathrm{ml})$ sufficient to dampen the lens paper was added. Explants were cultured in serum-free Leibovitz L-15 medium according to the technique described previously [27, 28]. After a 30 -min stabilisation period, the medium was replaced with fresh medium containing a final amount of $1.0 \mu \mathrm{mol} / \mathrm{ml}$ unlabelled oleic acid with $0.018 \mathrm{MBq}\left[{ }^{14} \mathrm{C}\right]$-oleic acid (specific activity, $2.11 \mathrm{GBq} / \mathrm{mmol}$; GE Healthcare Bio-Sciences, Baie D'Urfé, QC, Canada) in a micellar mixture $(6.6 \mathrm{mmol} / \mathrm{l}$ sodium taurocholate, $1 \mathrm{mmol} / \mathrm{l}$ oleic acid, $0.5 \mathrm{mmol} / 1 \mathrm{monoolein}, 0.1 \mathrm{mmol} / \mathrm{l}$ cholesterol, and $0.6 \mathrm{mmol} / 1$ phosphatidylcholine) [29]. Intestinal explants from Psammomys obesus gerbils were cultured for $3 \mathrm{~h}$. After this incubation period, tissue integrity was confirmed by morphological examination at the light microscopy level and biochemical (sucrase activity) studies (results not shown).

\section{Lipid and lipoprotein analyses}

Aliquots of explant homogenates and their respective incubation media were lipid-extracted with $2: 1(\mathrm{vol} / \mathrm{vol})$ chloroform : methanol (10-13). Small amounts of lipid standards were added to the samples before the separation of individual lipid classes by one-dimensional thin-layer chromatography using silica gel (Eastman Kodak, Rochester, NY, USA) as described previously [27, 29]. The non-polar solvent system was 80:20:3 ( $\mathrm{vol} / \mathrm{vol} / \mathrm{vol}$ ) hexane:diethylether:glacial acetic acid. The radioactivity of the separated fractions was measured in a liquid scintillation spectrometer (Beckman Instruments, Montreal, QC, Canada). Quenching was corrected using computerised curves generated with external standards. An aliquot of the tissue homogenate was used for protein determinations [27-30]. For the determination of secreted lipoproteins, the medium supplemented with anti-proteases was first mixed with a plasma lipid carrier $(2: 0.6 \mathrm{vol} / \mathrm{vol})$ to efficiently isolate the newly synthesised triglyceride-rich lipoproteins. The latter were then isolated at a density of $1.006 \mathrm{~g} / \mathrm{ml}$ by spinning at $100,000 \mathrm{~g}$ for $2.26 \mathrm{~h}$ with a tabletop ultracentrifuge (Beckman Instruments) as described previously $[27,28]$.

\section{Lipid carrier}

To provide a carrier for lipoproteins synthesised in vitro, postprandial plasma was obtained from healthy volunteers $3 \mathrm{~h}$ after the ingestion of fat $\left(50 \mathrm{~g} / 1.73 \mathrm{~m}^{2}\right)$ as described previously [27, 28].

De novo apolipoprotein synthesis

After incubation with $11.1 \mathrm{MBq} / \mathrm{ml}\left[{ }^{35} \mathrm{~S}\right]$-methionine (specific activity $39.5 \mathrm{TBq} / \mathrm{mmol}$, GE Healthcare Bio-Sciences), jejunal explants were washed (three times) with methioninefree Leibovitz medium and homogenised in PBS containing $1 \%(\mathrm{wt} / \mathrm{vol})$ Triton X-100, methionine ( $2 \mathrm{mmol} / \mathrm{l})$, phenylmethylsulfonyl fluoride $(1 \mathrm{mmol} / \mathrm{l})$, and benzamidine $(1 \mathrm{mmol} / \mathrm{l})$. The homogenates were centrifuged $\left(4^{\circ} \mathrm{C}\right)$ at $105,000 \mathrm{~g}$ for $60 \mathrm{~min}$ and supernatants subsequently reacted with excess apo B polyclonal antibodies for $18 \mathrm{~h}$ at $4^{\circ} \mathrm{C}$. Anti-Psammomys obesus apo B antiserum was raised in rabbits. Pansorbin was then added, and the mixture was reincubated at $20^{\circ} \mathrm{C}$ for $60 \mathrm{~min}$. The immunoprecipitates were washed extensively and analysed by a linear 4 to $20 \%$ acrylamide gradient preceded by a $3 \%$ stacking gel as described previously [30]. Apo B-48 bands on gels were sectioned and counted after overnight incubation at $20^{\circ} \mathrm{C}$ with $1 \mathrm{ml} \mathrm{BTS-450} \mathrm{(Beckman} \mathrm{Instruments)} \mathrm{and} 10 \mathrm{ml}$ liquid scintillation fluid (Ready Sol NA; Beckman Instruments).

Microsomal triglyceride transfer protein assays

Intestinal microsomes used as the source of microsomal triglyceride transfer protein (MTP) activity were isolated as described previously [31, 32]. MTP activity was determined by the transfer of radiolabelled triacylglycerol from 
donor small unilamellar vesicles $(40 \mathrm{nmol}$ egg phosphatidylcholine, $0.08 \mathrm{nmol}\left[{ }^{14} \mathrm{C}\right]$-triacylglycerol, and $2 \mathrm{nmol}$ cardiolipin) to acceptor small unilamellar vesicles $(240 \mathrm{nmol}$ egg phosphatidylcholine and $0.48 \mathrm{nmol}$ triacylglycerol) at $37^{\circ} \mathrm{C}$ for $1 \mathrm{~h}$. This assay has been described in detail elsewhere [31,32]. Lipid transfer activity is expressed as a percentage of triglyceride transfer per unit time under verified linear assay conditions (first-order kinetics). To assess the presence of MTP and evaluate its mass, homogenates of intestinal tissue were prepared for western blotting as described previously [31, 32]. Proteins were separated on a 4 to $20 \%$ gradient SDS-PAGE and electroblotted on to nitrocellulose membranes. Non-specific binding sites of the membranes were blocked using defatted milk proteins, followed by the addition of primary antibodies directed against MTP. The relative amount of primary antibody was detected with horseradish peroxidase-conjugated secondary antibody. Blots were developed, and the mass of MTP was quantitated using an HP Scanjet scanner equipped with a transparency adapter and HP scanner software.

\section{Enzymatic activities}

Activities of monoacylglycerol acyltransferase (MGAT) and diacylglycerol acyltransferase (DGAT) were determined in microsomes as reported by Coleman [33].

\section{Biochemical analyses}

Plasma glucose was determined by an enzymatic glucose analyser, and insulin levels were assessed by radioimmunoassay using a human primary antibody (Phadesph; Kabi Pharmacia Diagnostics, Uppsala, Sweden). Plasma triglyceride and cholesterol levels were measured colorimetrically (Boehringer Mannheim, Montreal, QC, Canada).

\section{Statistical analysis}

To assess differences in the parameters studied, data were statistically analysed by ANOVA. Differences between mean values were evaluated by Student's two tailed $t$-test.

\section{Results}

A significant difference in the pattern of body weight, glucose levels and insulin concentrations was evident following the consumption of $n-3$ FA and control alimentary regimens (Fig. 1). Consumption of a high- $n-3$-FA diet resulted in decreased weight gain in normoinsulinaemic/ normoglycaemic (Group A), hyperinsulinaemic/normogly- caemic (Group B) and hyperinsulinaemic/hyperglycaemic (Group C) animals (Fig. 1) in comparison with control diet intervention. Furthermore, Group B animals on n-3enriched food were characterised by reduced hyperinsulinaemia, which usually appears to compensate for both peripheral and hepatic insulin resistance as evidenced by the persistence of normoglycaemia. Diabetic Group C animals developed less hyperglycaemia and hyperinsulinaemia in response to diet enriched by $n-3$ FA. Moreover, administration of the $n-3$ FA diet reduced the hypertriglyceridaemia and hypercholesterolaemia characterising Groups B and C (Fig. 2). Thus this first series of experiments shows that the $n-3$ FA diet improved various abnormalities associated with the metabolic syndrome, including increased body weight, insulin resistance and dyslipidaemia.

We next studied intestinal lipogenesis in the experimental animal groups. As illustrated in Fig. 3, the incorporation of ${ }^{3} \mathrm{H}_{2} \mathrm{O}$ into total lipids in cultured jejunal explants was lower in all the animal groups on the $n-3$ regimen, albeit only the differences in Group $\mathrm{C}$ reached statistical
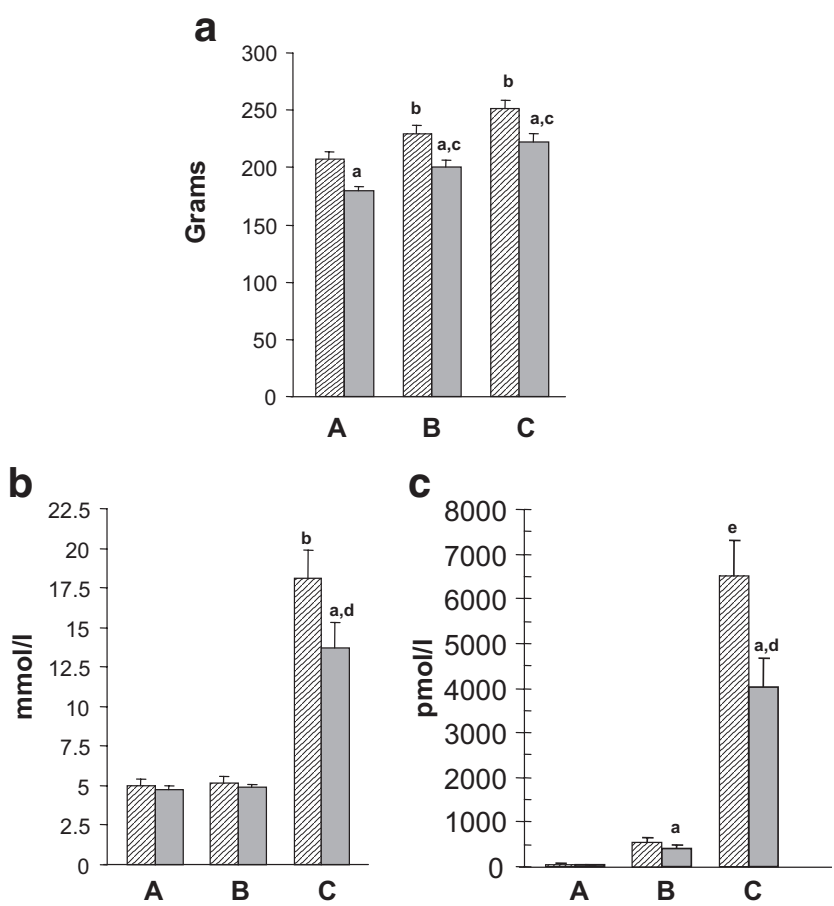

Fig. 1 Effects of $n$-3 fatty acid (FA) dietary intake on body weight (a), plasma glucose (b) and insulin concentrations (c) in Psammomys obesus gerbils. Upon weaning, the animals were randomly maintained on either a control or an $n$-3-FA rich diet. Animals were then killed and categorised according to biochemical characterisation of the blood samples as follows: Group A (A), normoglycaemic/normoinsulinaemic; Group B (B), normoglycaemic/hyperinsulinaemic; Group C (C), hyperglycaemic/hyperinsulinaemic. Hatched bars, basal diet; grey bars, $n-3$ FA diet. Values are means \pm SEM for $n=10$ (Group A), $n=8$ (Group B), and $n=7$ (Group C). ${ }^{\mathrm{a}}, p<0.05$ vs basal diet; ${ }^{\mathrm{b}}, p<0.05$ vs A on basal diet; $;, p<0.05$ vs A on $n-3$ FA diet; ${ }^{\mathrm{d}}, p<0.01$ vs A and B on $n$ -3 FA diet; ${ }^{\mathrm{e}}, p<0.01$ vs A and $\mathrm{B}$ on basal diet 
significance. To assess the incorporation of this radiolabelled precursor into major lipid species, we cultured jejunal explants with $\left[{ }^{14} \mathrm{C}\right]$-oleic acid. Figure 4 documents significant lipid changes in tissue homogenates and media. In fact, the administration of the $n-3$ FA diet to Psammomys obesus gerbils reduced the intestinal synthesis and secretion of triglycerides, phospholipids and cholesteryl esters. Interestingly, the decrease in Groups B and C was more substantial than that in Group A and was associated with the decline of MGAT and DGAT activities (Fig. 5).

To determine whether the $n-3$ FA diet affected the ability of the intestine to produce triglyceride-rich lipoproteins, $\left[{ }^{14} \mathrm{C}\right]$-oleic acid was added to jejunal explants obtained from the different animal groups (Fig. 6). The isolation of triglyceride-rich lipoproteins from media by ultracentrifugation and radioactivity measurement of lipoprotein fractions revealed that output was decreased in all groups administered the $n-3$ alimentary regimen, with the reductions in Groups B and C being greater.

The next step was to explore whether the $n-3$ FA diet altered apo B-48 biogenesis. For this purpose, jejunal
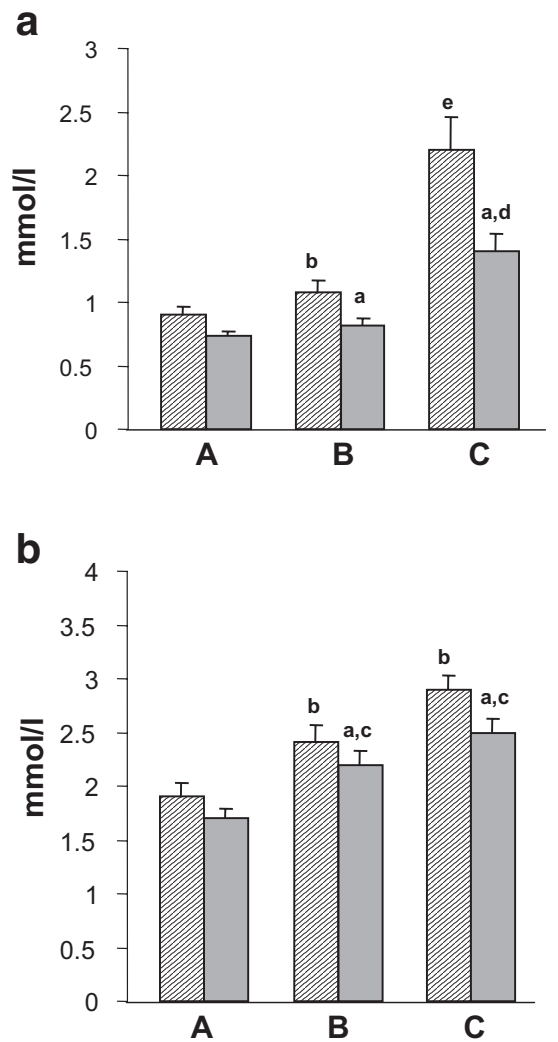

Fig. 2 Plasma lipids in Psammomys obesus gerbils. Levels of triglycerides (a) and total cholesterol (b) were determined after animals were killed. Animal groups: Group A (A), normoglycaemic/ normoinsulinaemic; Group B (B), normoglycaemic/hyperinsulinaemic; Group C (C), hyperglycaemic/hyperinsulinaemic. Hatched bars, basal diet; grey bars, $n-3$ FA diet. ${ }^{\mathrm{a}}, p<0.05$ vs basal diet; ${ }^{\mathrm{b}}, p<0.05$ vs A on basal diet; ${ }^{\mathrm{c}}, p<0.05$ vs A on $n-3$ FA diet; ${ }^{\mathrm{d}}, p<0.01$ vs A and $\mathrm{B}$ on $n-3$ FA diet; ${ }^{\mathrm{e}}, p<0.01$ vs $\mathrm{A}$ and $\mathrm{B}$ on basal diet explants were cultured with $\left[{ }^{35} \mathrm{~S}\right]$-methionine. Jejunal explants derived from insulin-resistant and diabetic animals fed with $n$-3 FA were less prone to synthesise apo B-48 (Fig. 7a). In contrast, no alterations were detected in apo B48 mRNA (data not shown). Since proteasomal degradation of apo B plays a regulatory role in the early stages of apo B biosynthesis, we wondered whether this pathway is triggered by $n-3$ FA. The addition of $N$-acetyl-leucylnorleucinat and lactacystin as proteasomal inhibitors [18] suppressed the differences observed among groups, regardless of dietary regimen (Fig. 7b). We also assessed protein expression of MTP, which is required for apo B-48 lipoprotein assembly and secretion, in intestinal tissues from the three groups of Psammomys obesus gerbils. No significant modifications were seen in any of the experimental animal groups (results not shown), possibly suggesting that $n$-3 FA do not inhibit MTP expression and decrease apo B-48 synthesis and triglyceride-rich lipoproteins by this pathway.

\section{Discussion}

Psammomys obesus (sand rat) is a well-established model of type 2 diabetes and obesity. A rodent found in desert areas, it has normal metabolic parameters when on a diet of relatively low caloric density. When the same animals are fed standard chow, they acquire most of the features of the human metabolic syndrome: obesity, hyperglycaemia, hyperinsulinaemia, hyperleptinaemia and dyslipidaemia [26, 34, 35]. Referenced against normoglycaemic and normoinsulinaemic Psammomys obesus gerbils (Group A), a high proportion of Group B animals displayed hyperinsulinaemia, which appears to compensate

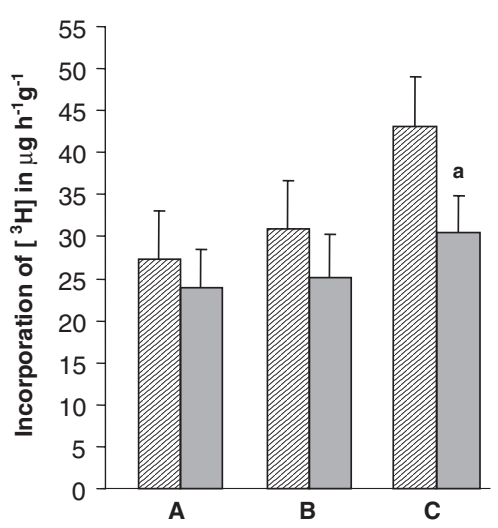

Fig. 3 Intestinal lipogenesis in Psammomys obesus groups. A Group A, normoglycaemic/normoinsulinaemic; B Group B, normoglycaemic/hyperinsulinaemic; $\mathrm{C}$ Group $\mathrm{C}$, hyperglycaemic/hyperinsulinaemic. Hatched bars, basal diet; grey bars, $n-3$ FA diet. The rate of lipogenesis was measured with ${ }^{3} \mathrm{H}_{2} \mathrm{O}$ and determined by liquid scintillation counting. Values are means \pm SEM for $n=5$ per group and per diet. ${ }^{\mathrm{a}}, p<0.05$ vs basal diet 

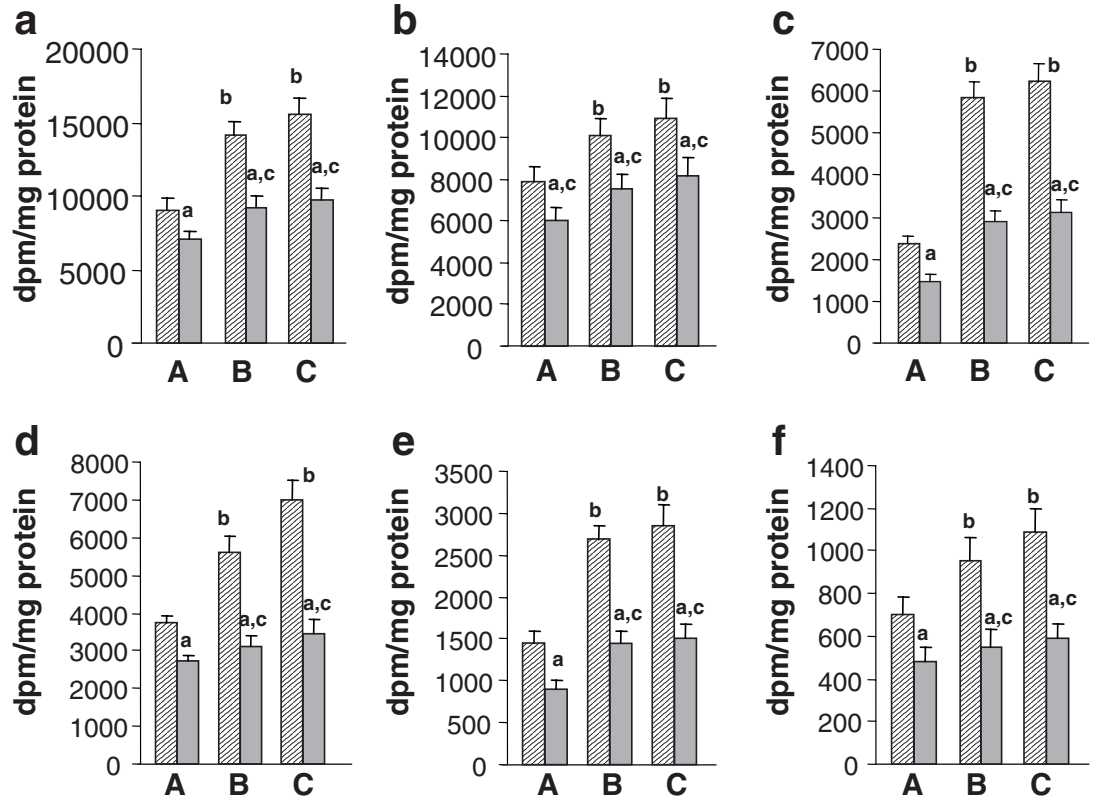

Fig. 4 De novo lipid synthesis and secretion in Psammomys obesus intestinal explants from A, Group A, normoglycaemic/normoinsulinaemic; B, Group B, normoglycaemic/hyperinsulinaemic; C, Group $\mathrm{C}$, hyperglycaemic/hyperinsulinaemic. Hatched bars, basal diet; grey bars, $n$-3 FA diet. Jejunal explants were incubated with $\left[{ }^{14} \mathrm{C}\right]$-oleic acid substrate for $3 \mathrm{~h}$. Triglycerides (a,d), phospholipids (b,e), cholesteryl esters $(\mathbf{c}, \mathbf{f})$ were then extracted with chloroform : methanol $(2: 1, v / v)$ from tissue homogenates $(\mathbf{a}, \mathbf{b}, \mathbf{c})$ and media $(\mathbf{d}, \mathbf{e}, \mathbf{f})$, isolated by thin-layer chromatography, and quantitated as described in Materials and methods. Values represent means \pm SEM of three separate experiments. ${ }^{\mathrm{a}}, p<0.05$ vs basal diet; ${ }^{\mathrm{b}}, p<0.05$ vs $\mathrm{A}$ on basal $\operatorname{diet}^{\mathrm{c}}, p<0.05$ vs A on $n$-3 FA diet for both peripheral and hepatic insulin resistance as evidenced by the persistence of normoglycaemia [26]. In Group C, glycaemia can no longer be compensated for, in spite of the extraordinary elevation in plasma insulin. Recently, we demonstrated that the development of insulin-resistant and type 2 diabetic states led to intestinal oversecretion of apo B-48 lipoproteins in Psammomys obesus gerbils [18]. The results of the present investigation indicate the beneficial effects of diets enriched with $n-3$ FA on body weight, hyperglycaemia, hyperinsulinaemia and hyperlipidaemia. Additionally, n-3 FA intake lowered lipogenesis, lipid esterification and de novo apo B-48 synthesis, partially abolishing the overproduction of intestinal triglyceride-rich lipoproteins. While $n-3$ FA administration did not affect expression of the genes encoding DGAT1, apo B, and MTP, it was able to induce Dgat2 mRNA and to promote proteasomal degradation of apo B. Our data point out the complex and multifaceted actions of $n-3$ FA.

Several clinical studies in the 1980s and early 1990s reported adverse effects on blood glucose control and insulin activity in subjects with type 2 diabetes who consumed large amounts of fish oil $[36,37]$. These deleterious effects may be attributable to the high doses used, e.g. $10 \mathrm{~g}$ fish oil per day or more. Recent studies using low doses of $n-3$ LCPUFA, ranging from 1 to $2 \mathrm{~g} / \mathrm{day}$, did not report deteriorations in glucose control [22, 38-40]. Our experi- ments clearly showed that moderate $n-3$ FA intake improved hyperglycaemia and hyperinsulinaemia in Psammomys obesus gerbils.

Postprandial triglyceride-rich lipoproteins and especially chylomicron remnants have been implicated as risk factors for atherosclerosis and progression of coronary artery disease [41-45]. Although the physiological basis of metabolic dyslipidaemia appears to be hepatic overproduction of apoB-containing VLDL particles [46, 47], emerging evidence suggests that intestinal overproduction of apo-B-48-containing lipoproteins in insulin-resistant states may be an important contributor to the elevation of circulating triglyceride-rich lipoproteins $[17,18,48]$. The present study demonstrated that intestinal cells, like hepatocytes [49, 50], responded to $n-3$ FA and decreased the output of triglyceride-rich lipoproteins by lowering the synthesis of apo B-48 and by inhibiting the new formation of lipids. The suppression of apo B-48 was not due to changes in apo B transcription, since $A p o b 48$ mRNA levels were unaffected. It also did not involve MTP, an essential component in apoB-48-containing lipoprotein assembly and secretion [51]. One possible explanation may be the limiting availability of triglycerides, phospholipids and cholesteryl esters that are known to protect apo B-48 from proteasome degradation. The addition of a proteasome inhibitor to the culture of jejunal explants from insulin-resistant and diabetic animals enhanced the production of apo B-48. It seems therefore that, 
a

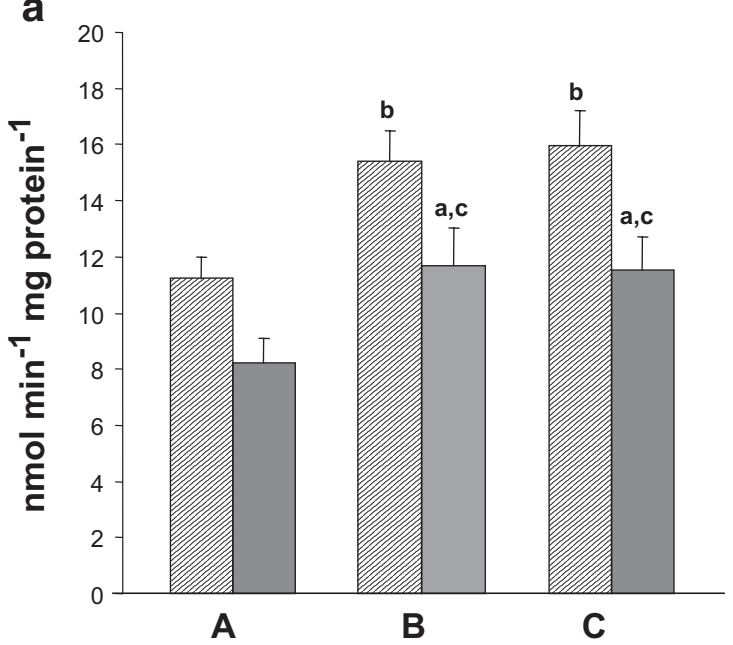

b

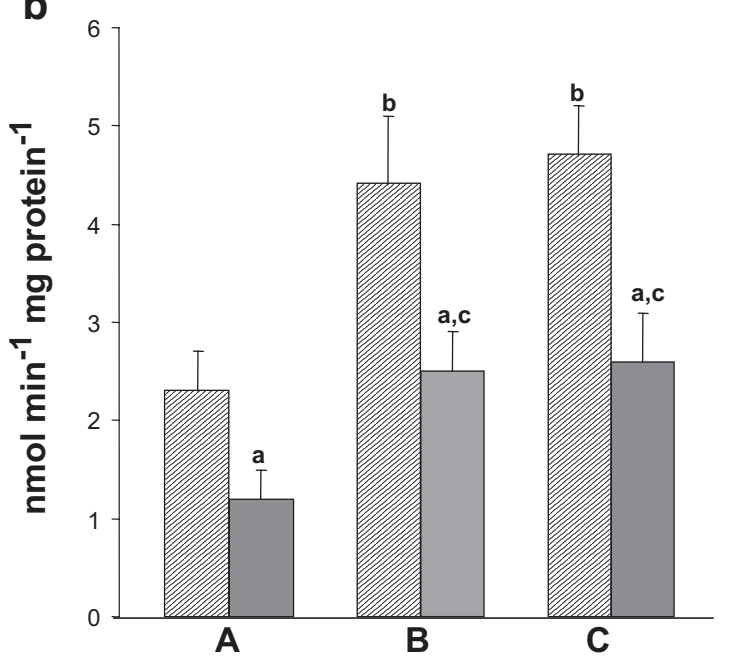

Fig. 5 MGAT (a) and DGAT (b) activity in the small intestine of Psammomys obesus gerbils. Microsomes were prepared from the intestinal tissue of the three gerbil groups (see legend, Figs 1, 2, 3 and 4) and assayed for the two enzymes. Hatched bars, basal diet; grey bars, $n-3$ FA diet. Data represent means \pm SEM for $n=5$ (Group A), $n=4$ (Group B) and $n=4$ (Group C). ${ }^{\mathrm{a}}, p<0.05$ vs basal diet; ${ }^{\mathrm{b}}, p<0.05$ vs A on basal diet; ${ }^{\mathrm{c}}, p<0.05$ vs A on $n-3$ FA diet

in Psammomys obesus gerbils affected by insulin resistance and diabetes, dietary $n-3$ FA enhanced apo B secretion via the intracellular proteasome-mediated posttranslational downregulation apo B degradation pathway.

MGAT catalyses the synthesis of diacylglycerols from monoacylglycerols and long-chain fatty acyl coenzyme A esters, the first step in the monoacylglycerol pathway contributing to glycerolipid synthesis in the intestinal mucosa [52]. This action is followed by the acylation of diacylglycerols by DGAT to produce triacylglycerols. The current experiments confirmed previous data showing high levels of MGAT and DGAT activity, which is consistent with the remarkable capacity of the small intestine to produce triacylglycerol in an insulin-resistant and diabetic state. The administration of a diet enriched in $n-3$ FA

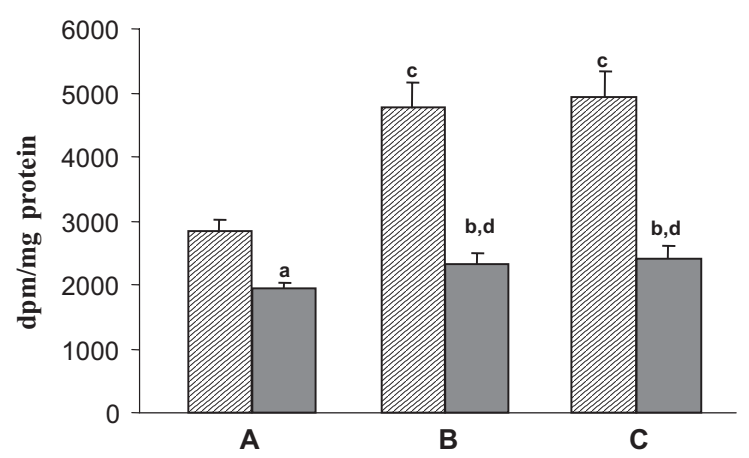

Fig. 6 Production of triglyceride-rich lipoproteins by jejunal explants of Psammomys obesus gerbils (groups, see legend, Figs 1, 2, 3 and 4). Hatched bars, basal diet; grey bars, $n$-3 FA diet. Jejunal explants were cultured in the presence of $\left[{ }^{14} \mathrm{C}\right]$-oleic acid. After $3 \mathrm{~h}$ of incubation, tryglyceride-rich lipoproteins were isolated by ultracentrifugation. Values are means \pm SEM of three separate experiments. ${ }^{\mathrm{a}}, p<0.05 \mathrm{vs}$ basal diet; ${ }^{\mathrm{b}}, p<0.01$ vs basal diet; ${ }^{\mathrm{c}}, p<0.01$ vs A on basal diet; ${ }^{\mathrm{d}}$, $p<0.01$ vs A on $n-3$ FA diet

resulted in lower activities of these enzymes, thereby reflecting the favourable action of fish oil on intestinal mucosa, which led to decreased lipid production under insulin resistance and diabetic conditions.

In conclusion, our data revealed that $n-3 \mathrm{FA}$ lowered the hyperglycaemia, hyperinsulinaemia and dyslipidaemia that characterise insulin-resistant and diabetic Psammomys
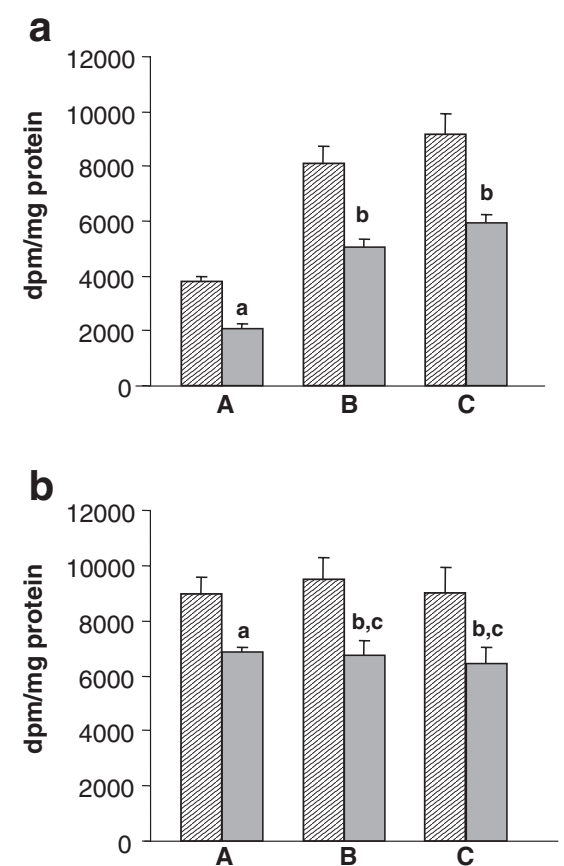

Fig. 7 Apo B-48 synthesis in Psammomys obesus intestinal explants (groups, see legend, Figs 1, 2, 3 and 4). Hatched bars, basal diet; grey bars, $n-3$ FA diet. Jejunal explants were incubated for $3 \mathrm{~h}$ with methionine-free medium containing $\left[{ }^{35} \mathrm{~S}\right]$-methionine in the presence (a) or absence (b) of proteasome inhibitors [ $N$-acetyl-leucyl-norleucinat $(40 \mu \mathrm{g})$ and lactacystin $(1 \mu \mathrm{mol} / \mathrm{l})]$. Apo B-48 was then immunoprecipitated and analysed by gel electrophoresis. Values represent means $\pm \mathrm{SEM}$ of three separate experiments. ${ }^{\mathrm{a}}, p<0.05$ vs basal diet; ${ }^{\mathrm{b}}, p<0.01$ vs basal diet; ${ }^{\mathrm{c}}, p<0.01$ vs A on n-3 FA diet 
obesus gerbils. The lipid improvements were chiefly due to a reduction in apo-B-48-containing lipoprotein secretion, achieved via the regulation of de novo lipid and apo B-48 production. The reduced secretion of apo B that was essentially mediated by post-translational degradation of the newly synthesised protein and occurred subsequently to limited lipid availability may explain the intestinal fat transport-lowering effects of PUFA.

Acknowledgements This study was supported by research grants from the Canadian Diabetes Association (CDA \#1633) and the Canadian Institutes of Health Research (CIHR \#MT-10584).

\section{References}

1. Jeppesen J, Hein HO, Suadicani P, Gyntelberg F (1998) Triglyceride concentration and ischemic heart disease: an eightyear follow-up in the Copenhagen Male Study. Circulation 97:1029-1036

2. Castelli WP (1992) Epidemiology of triglycerides: a view from Framingham. Am J Cardiol 70:3H-9H

3. Assmann G, Schulte H, Cullen P (1997) New and classical risk factors - the Munster heart study (PROCAM). Eur J Med Res 2:237-242

4. Austin MA (1991) Plasma triglyceride and coronary heart disease. Arterioscler Thromb 11:2-14

5. Hokanson JE, Austin MA (1996) Plasma triglyceride level is a risk factor for cardiovascular disease independent of high-density lipoprotein cholesterol level: a meta-analysis of population-based prospective studies. J Cardiovasc Risk 3:213-219

6. Ericsson CG, Hamsten A, Nilsson J, Grip L, Svane B, de Faire U (1996) Angiographic assessment of effects of bezafibrate on progression of coronary artery disease in young male postinfarction patients. Lancet 347:849-853

7. Miller M, Seidler A, Moalemi A, Pearson TA (1998) Normal triglyceride levels and coronary artery disease events: the Baltimore Coronary Observational Long-Term Study. J Am Coll Cardiol 31:1252-1257

8. Karpe F, Steiner G, Uffelman K, Olivecrona T, Hamsten A (1994) Postprandial lipoproteins and progression of coronary atherosclerosis. Atherosclerosis 106:83-97

9. Patsch JR, Miesenbock G, Hopferwieser T et al (1992) Relation of triglyceride metabolism and coronary artery disease. Studies in the postprandial state. Arterioscler Thromb 12:1336-1345

10. Groot PH, van Stiphout WA, Krauss XH et al (1991) Postprandial lipoprotein metabolism in normolipidemic men with and without coronary artery disease. Arterioscler Thromb 11:653-662

11. Garcia MJ, McNamara PM, Gordon T, Kannel WB (1974) Morbidity and mortality in diabetics in the Framingham population. Sixteen year follow-up study. Diabetes 23:105-111

12. Haffner SM, Lehto S, Ronnemaa T, Pyorala K, Laakso M (1998) Mortality from coronary heart disease in subjects with type 2 diabetes and in nondiabetic subjects with and without prior myocardial infarction. N Engl J Med 339:229-234

13. Gotto AM Jr (1998) Triglyceride: the forgotten risk factor. Circulation 97:1027-1028

14. Marsh JB (2003) Lipoprotein metabolism in obesity and diabetes: insights from stable isotope kinetic studies in humans. Nutr Rev $61: 363-375$
15. Taskinen MR (2001) Pathogenesis of dyslipidemia in type 2 diabetes. Exp Clin Endocrinol Diabetes 109(Suppl 2):S180-S188

16. Syvanne M, Hilden H, Taskinen MR (1994) Abnormal metabolism of postprandial lipoproteins in patients with non-insulindependent diabetes mellitus is not related to coronary artery disease. J Lipid Res 35:15-26

17. Haidari M, Leung N, Mahbub F et al (2002) Fasting and postprandial overproduction of intestinally derived lipoproteins in an animal model of insulin resistance. Evidence that chronic fructose feeding in the hamster is accompanied by enhanced intestinal de novo lipogenesis and ApoB48-containing lipoprotein overproduction. J Biol Chem 277:31646-31655

18. Zoltowska M, Ziv E, Delvin E et al (2003) Cellular aspects of intestinal lipoprotein assembly in Psammomys obesus: a model of insulin resistance and type 2 diabetes. Diabetes 52:2539-2545

19. Kromhout D, Bosschieter EB, de Lezenne CC (1985) The inverse relation between fish consumption and 20-year mortality from coronary heart disease. N Engl J Med 312:1205-1209

20. von Schacky C (2000) n-3 fatty acids and the prevention of coronary atherosclerosis. Am J Clin Nutr 71:224S-227S

21. Harris WS (1989) Fish oils and plasma lipid and lipoprotein metabolism in humans: a critical review. J Lipid Res 30:785-807

22. Sirtori CR, Crepaldi G, Manzato E et al (1998) One-year treatment with ethyl esters of $n-3$ fatty acids in patients with hypertriglyceridemia and glucose intolerance: reduced triglyceridemia, total cholesterol and increased HDL-C without glycemic alterations. Atherosclerosis 137:419-427

23. Yosefy C, Viskoper JR, Laszt A et al (1999) The effect of fish oil on hypertension, plasma lipids and hemostasis in hypertensive, obese, dyslipidemic patients with and without diabetes mellitus. Prostaglandins Leukot Essent Fatty Acids 61:83-87

24. Glauber H, Wallace P, Griver K, Brechtel G (1988) Adverse metabolic effect of omega-3 fatty acids in non-insulin-dependent diabetes mellitus. Ann Intern Med 108:663-668

25. Kasim SE, Stern B, Khilnani S, McLin P, Baciorowski S, Jen KL (1988) Effects of omega-3 fish oils on lipid metabolism, glycemic control, and blood pressure in type II diabetic patients. J Clin Endocrinol Metab 67:1-5

26. Zoltowska M, Ziv E, Delvin E et al (2001) Circulating lipoproteins and hepatic sterol metabolism in Psammomys obesus prone to obesity, hyperglycemia and hyperinsulinemia. Atherosclerosis 157:85-96

27. Levy E, Thibault L, Menard D (1992) Intestinal lipids and lipoproteins in the human fetus: modulation by epidermal growth factor. J Lipid Res 33:1607-1617

28. Levy E, Menard D, Delvin E et al (2001) The polymorphism at codon 54 of the FABP2 gene increases fat absorption in human intestinal explants. J Biol Chem 276:39679-39684

29. Levy E, Marcel Y, Deckelbaum RJ et al (1987) Intestinal apoB synthesis, lipids, and lipoproteins in chylomicron retention disease. J Lipid Res 28:1263-1274

30. Levy E, Sinnett D, Thibault L, Nguyen TD, Delvin E, Menard D (1996) Insulin modulation of newly synthesized apolipoproteins B-100 and B-48 in human fetal intestine: gene expression and mRNA editing are not involved. FEBS Lett 393:253-258

31. Levy E, Stan S, Delvin E et al (2002) Localization of microsomal triglyceride transfer protein in the Golgi: possible role in the assembly of chylomicrons. J Biol Chem 277:16470-16477

32. Slight I, Bendayan M, Malo C, Delvin E, Lambert M, Levy E (2004) Identification of microsomal triglyceride transfer protein in intestinal brush-border membrane. Exp Cell Res 300:11-22

33. Coleman RA (1992) Diacylglycerol acyltransferase and monoacylglycerol acyltransferase from liver and intestine. Methods Enzymol 209:98-104

34. Angulo P (2002) Nonalcoholic fatty liver disease. N Engl J Med 346:1221-1231 
35. Kalman R, Adler JH, Lazarovici G, Bar-On H, Ziv E (1993) The efficiency of sand rat metabolism is responsible for development of obesity and diabetes. J Basic Clin Physiol Pharmacol 4:57-68

36. Borkman M, Chisholm DJ, Furler SM et al (1989) Effects of fish oil supplementation on glucose and lipid metabolism in NIDDM. Diabetes 38:1314-1319

37. Vessby B (1989) n-3 fatty acids and blood glucose control in diabetes mellitus. J Intern Med 731(Suppl):207-210

38. Westerveld HT, de Graaf JC, van Breugel HH et al (1993) Effects of low-dose EPA-E on glycemic control, lipid profile, lipoprotein (a), platelet aggregation, viscosity, and platelet and vessel wall interaction in NIDDM. Diabetes Care 16:683-688

39. Axelrod L, Camuso J, Williams E, Kleinman K, Briones E, Schoenfeld D (1994) Effects of a small quantity of omega-3 fatty acids on cardiovascular risk factors in NIDDM. A randomized, prospective, double-blind, controlled study. Diabetes Care 17:37-44

40. Luo J, Rizkalla SW, Vidal H et al (1998) Moderate intake of $n-3$ fatty acids for 2 months has no detrimental effect on glucose metabolism and could ameliorate the lipid profile in type 2 diabetic men. Results of a controlled study. Diabetes Care 21:717-724

41. Yu KC, Cooper AD (2001) Postprandial lipoproteins and atherosclerosis. Front Biosci 6:D332-D354

42. McNamara JR, Shah PK, Nakajima K et al (2001) Remnant-like particle (RLP) cholesterol is an independent cardiovascular disease risk factor in women: results from the Framingham Heart Study. Atherosclerosis 154:229-236

43. Karpe F, Boquist S, Tang R, Bond GM, de Faire U, Hamsten A (2001) Remnant lipoproteins are related to intima-media thickness of the carotid artery independently of LDL cholesterol and plasma triglycerides. J Lipid Res 42:17-21
44. Doi H, Kugiyama K, Oka H et al (2000) Remnant lipoproteins induce proatherothrombogenic molecules in endothelial cells through a redox-sensitive mechanism. Circulation 102:670-676

45. Boquist S, Ruotolo G, Tang R et al (1999) Alimentary lipemia, postprandial triglyceride-rich lipoproteins, and common carotid intima-media thickness in healthy, middle-aged men. Circulation 100:723-728

46. Adeli K, Taghibiglou C, Van Iderstine SC, Lewis GF (2001) Mechanisms of hepatic very low-density lipoprotein overproduction in insulin resistance. Trends Cardiovasc Med 11:170-176

47. Carpentier A, Taghibiglou C, Leung N et al (2002) Ameliorated hepatic insulin resistance is associated with normalization of microsomal triglyceride transfer protein expression and reduction in very low density lipoprotein assembly and secretion in the fructose-fed hamster. J Biol Chem 277:28795-28802

48. Lewis GF, Naples M, Uffelman K, Leung N, Szeto L, Adeli K (2004) Intestinal lipoprotein production is stimulated by an acute elevation of plasma free fatty acids in the fasting state: studies in insulin-resistant and insulin-sensitized Syrian golden hamsters. Endocrinology 145:5006-5012

49. Nestel PJ, Connor WE, Reardon MF, Connor S, Wong S, Boston R (1984) Suppression by diets rich in fish oil of very low density lipoprotein production in man. J Clin Invest 74:82-89

50. Harris WS (1997) n-3 fatty acids and serum lipoproteins: human studies. Am J Clin Nutr 65:1645S-1654S

51. Wetterau JR, Aggerbeck LP, Bouma ME et al (1992) Absence of microsomal triglyceride transfer protein in individuals with abetalipoproteinemia. Science 258:999-1001

52. Lehner R, Kuksis A (1996) Biosynthesis of triacylglycerols. Prog Lipid Res 35:169-201 